\title{
Le sonnet 130 de Shakespeare ou le blason mis à nu
}

\author{
NATALIE ROULON \\ Université de Strasbourg
}

\begin{abstract}
Shakespeare's sonnet 130 is sometimes read as an anti-blazon, and therefore as a misogynist text. Drawing on a large number of Renaissance poems, I show that this is a misreading of the sonnet which, far from presenting the Dark Lady in satirical fashion, pays her an unconventional tribute. Shakespeare seemingly discards worn out metaphors, the better to throw light on their arbitrary nature. Rather than disparaging his Lady, he offers a witty parody of the traditional Petrarchan representation of women : rather than an anti-blazon, sonnet 130 is best defined as a metablason.
\end{abstract}

Le sonnet 130 de Shakespeare est parfois lu comme un antiblason et, par conséquent, comme un texte misogyne. En mappuyant sur un grand nombre de poèmes de la Renaissance, je montre qu'il s'agit là d'une interprétation erronée du sonnet qui, loin de présenter la Dame brune sur un mode satirique, lui rend un hommage peu conventionnel. Shakespeare fait mine décarter les métaphores éculées de façon à mieux mettre en lumière leur caractère arbitraire. Plutôt que de dénigrer sa Dame, il propose une parodie spirituelle de la représentation des femmes propre à la tradition pétrarquiste: ce n'est pas la catégorie d'antiblason mais celle de métablason qui correspond le mieux au sonnet 130.

\section{$\mathrm{L}$}

e sonnet 130, l'un des plus connus de la série consacrée à la Dame brune, fait, comme un texte misogyne. D’après Frank Erik Pointner, par exemple, ce contreblason évoque une femme dont l'apparence physique est diamétralement opposée à celle de la Dame idéale de la descriptio pulchritudinis traditionnelle, une femme qui, par conséquent, est tout sauf belle ${ }^{1}$. Le texte ternirait donc l'image de la Dame. Qu'en est-il exactement?

L'objet de cet article est d'apporter une réponse à cette question en définissant le contexte littéraire dans lequel s'inscrit le poème, c'est-à-dire en montrant comment il se situe par rapport à la tradition pétrarquiste, et au blason en particulier. En effet, seule une étude comparative peut permettre d'évaluer la dette de Shakespeare à l'égard des sonnettistes élisabéthains et de leurs prédécesseurs français afin de saisir la spécificité de son geste poétique.

1. Erik F. Pointner, Bawdy and Soul : A Revaluation of Shakespeare's Sonnets (Heidelberg : Universitätsverlag Winter, 2003), p. 85 et 165. 
1 My mistress' eyes are nothing like the sun ;

Coral is far more red than her lips' red ;

If snow be white, why then her breasts are dun ;

If hairs be wires, black wires grow on her head.

5 I have seen roses damasked, red and white,

But no such roses see I in her cheeks,

And in some perfumes is there more delight

Than in the breath that from my mistress reeks.

9 I love to hear her speak, yet well I know

That music hath a far more pleasing sound.

I grant I never saw a goddess go ;

My mistress when she walks treads on the ground.

13 And yet, by heaven, I think my love as rare

As any she belied with false compare. ${ }^{2}$

Dès le XIII ${ }^{e}$ siècle, le rhétoricien anglais Geoffrey de Vinsauf avait fixé les règles de ce que l'on pourrait appeler le blason pré-marotique : pour décrire une femme, il convenait de louer les différentes parties de son corps de la tête aux pieds, ordre déjà suivi par la narratrice du cantique des cantiques pour l'éloge de son bien-aimé (5, 10-16). Dans sa Nova Poetria, Vinsauf donne un exemple d'effictio (description complète du corps humain) dont les principaux éléments se retrouveront dans la poésie pétrarquiste : les cheveux sont d'or; des lys ornent le front majestueux ; les yeux ont l'éclat des étoiles; semblable à l'aurore, le visage n'est ni rouge ni blanc mais les deux à la fois ; la bouche est charnue mais petite, l'haleine, parfumée ; c'est la blancheur, qu'elle soit celle de la neige, du lait ou du marbre, qui caractérise les dents, le menton, le cou et les seins ; les doigts sont effilés et les pieds, menus ${ }^{3}$. Texte normatif, la Nova Poetria fut un manuel de rhétorique très utilisé dans les écoles et universités européennes entre le XIII et le $\mathrm{XV}^{\mathrm{e}}$ siècle, d'où son influence sur la littérature du Moyen Âge et du début de la Renaissance ${ }^{4}$.

2. John Kerrigan, éd., The Sonnets and A Lovers' Complaint (London : Penguin, 1999), p. 141.

3. Ernest Gallo, The Poetria Nova and its Sources in Early Rhetorical Doctrine (The Hague, Paris : Mouton, 1971), p. 44-45.

4. Cf. Marjorie C. Woods, Classroom Commentaries : Teaching the Poetria Nova across Medieval and Renaissance Europe (Columbus : Ohio State University Press, 2010). 
On rencontre chez Pétrarque le prototype de ce qui, après Clément Marot, sera connu sous le nom de "blason anatomique du corps féminin $»^{5}$ : le sonnet 199 du Chansonnier est un blason de la main avant la lettre. Exilé à Ferrare en raison de ses sympathies protestantes, Marot, qui avait introduit en France le sonnet pétrarquiste ${ }^{6}$, lança la vogue du blason en 1535. Sous l'influence des strambottistes, et plus particulièrement d'Olimpo da Sassoferrato, qui s'adonnait à de « lestes peintures des charmes de ses maîtresses » ${ }^{7}$, il écrivit le «Blason du beau tetin », qui adresse un éloge au sein féminin, et dont voici les premiers vers : «Tetin refait, plus blanc qu'un œuf, / Tetin de satin blanc tout neuf, / Tetin qui fais honte à la rose, / Tetin plus beau que nulle chose $[\ldots] »^{8}$. Comparé à des matières précieuses comme le satin ou l'ivoire et à des végétaux odorants ou savoureux comme la rose, la fraise ou la cerise, le sein est isolé du corps de la femme et décrit sous de multiples facettes par un sujet masculin désirant - «[...] le blason de la dame est surtout un chant du désir du poète » rappelle Thomas Hunkeler 9 . À la différence du blason de Pétrarque, qui contient une référence au Ciel, le blason marotique est nettement profane.

En France, le « Blason du beau tetin » fut accueilli comme une exquise nouveauté : «Le roi François et sa cour applaudirent, quand le poème leur

5. Pour l'historique du blason, on se reportera utilement à l'étude d'Alison Saunders, The Sixteenth-Century Blason Poétique (Berne : Peter Lang, 1981).

6. Il a traduit des poèmes du Chansonnier, tant parce qu'il était fasciné par son auteur que pour plaire à François I Ier, qui vouait un culte à Pétrarque et invitait ses poètes à « pétrarquiser ». D’autre part, «C’est sans doute Pétrarque - l'Italie - qui pousse Marot, par mimétisme, à pratiquer le sonnet ». Gérard Defaux, éd., Euvres poétiques II (Paris : Bordas, 1993), p. 1193-1194.

7. Michel Simonin, éd., Dictionnaire des lettres françaises, XVI siècle (Paris : Fayard et Librairie Générale Française, 2001), p. 154. Si l'influence italienne sur le blason anatomique ne fait aucun doute pour J. Vianey, V.-L. Saulnier y voit, au contraire, le point d'aboutissement d'une longue tradition française. Pour une approche nuancée de cette question, voir l'ouvrage de Saunders, qui conclut que l'empreinte de Sassoferrato est manifeste sur le plan formel.

8. Jean-Clarence Lambert, éd., Blasons du corps féminins (Paris : Union Générale d'Éditions, 1996), p. 59-60.

9. Thomas Hunkeler, Le Vif du sens. Corps et poésie selon Maurice Scève (Genève : Droz, 2003), p. 84. Saunders explique que « [...] la description de la beauté féminine, telle que nous la trouvons dans les blasons anatomiques, est par essence subjective, inséparablement liée à la description de la réaction du poète-amant face à cette beauté ». " "La beauté que femme doibt avoir" : la vision du corps dans les blasons anatomiques » in Le Corps à la Renaissance, éd. J. Céard, M.-M Fontaine et J.-C. Margolin (Paris : Aux Amateurs de Livres, 1990), p. 46. 
parvint. [...] Tous ceux qui pratiquaient le beau langage se mirent d'emblée à blasonner ${ }^{10}$. Si le blason de Marot et ceux qui le suivirent ne sont pas des sonnets mais des poèmes à rimes plates, métriquement divers (octosyllabes ou décasyllabes) et de longueur variable, on peut néanmoins affirmer que bien des sonnets, ceux des membres de la Pléiade en particulier, n'auraient jamais existé sans eux.

Il convient de distinguer le blason du corps entier, qui en énumère les divers attraits et dont on trouve un seul exemple (anonyme) dans le recueil des Blasons anatomiques du corps féminin de $1543^{11}$, du blason qui isole une partie du corps, type dominant dans la production de Marot et de ses imitateurs ${ }^{12}$, tels que Maurice Scève ou Jacques Peletier du Mans. Cela dit, dans les deux types de blason s'effectue un morcellement du corps féminin que l'on peut rattacher à l'esthétique maniériste dont la France voit l'épanouissement à cette époque ${ }^{13}$. Le «Blason du beau tetin " "déclencha une spirale de rivalité mimétique »

\section{Lambert, p. 10.}

11. Version numérisée par le CÉSR de Tours, 2009, p. 53-54. On trouve un blason synthétique dans le Chansonnier à l'état embryonnaire : «Les yeux dont je parlai si ardemment, / et les bras et les mains, les pieds et le visage, / qui m'avaient éloigné tellement de moi-même [...] la chevelure d'or pur et brillant, bouclée, / et le scintillement de son rire angélique » (292, v. 1-6). Cela dit, de telles énumérations sont rares chez Pétrarque qui, en outre, ne respecte pas l'ordre établi par Vinsauf.

12. Dans l'édition de 1536, les blasons anatomiques apparaissent dans un ordre disparate : le sein est suivi du cheveu, qui est suivi du cœur, lui-même suivi de la cuisse, etc. Dans certaines éditions ultérieures comme celle de 1539, ils sont agencés de telle sorte que la hiérarchie prescrite par Vinsauf est respectée au niveau de la structure d'ensemble : les blasons des cheveux sont suivis du blason du front, lui-même suivi du blason du sourcil... jusqu'au blason du pied. Comme le suggère Nancy Vickers, c'est la norme descriptive conventionnelle qui s'est imposée : Nancy Vickers, " Members Only. Marot's Anatomical Blazons ", in The Body in Parts. Fantasies of Corporeality in Early Modern Europe, éd. D. Hillman et C. Mazzio (New York and London : Routledge, 1997), p. 9-10.

13. Dans La Poésie française et le maniérisme, 1546-1610?, Marcel Raymond rappelle que Fontainebleau est le foyer de l'art maniériste dans les années 1530-1585 (Genève : Droz, 1971), p. 8. Il explique que si l'âge baroque se caractérise par « [...] une vision unifiante, il n'en va pas de même à l'âge précédent, où l'on préfère une vision morcelante, l'œuvre ne se découvrant d'aucun point de vue dans sa totalité » (p. 21). Claude-Gilbert Dubois voit dans le blason «l'équivalent littéraire d'un médaillon » et le décrit encore comme " la reproduction en vers d'un portrait ou d'un détail pictural ». Il évoque la parenté stylistique du blason et des genres picturaux contemporains en mentionnant « le goût de l'accumulation des détails qui se trouvent à la fois dans les textes et tableaux maniéristes, la multiplicité l'emportant dans les deux cas sur l'unité ": Claude-Gilbert Dubois, Le Maniérisme (Paris : Presses Universtaires de France, 1979), p. 94. 
telle que «Chacun prit une cuisse, un soupir, une chevelure, un œil, une main, un cœur, une larme, une oreille, une langue, une voix $»^{14}$. Ce qui faisait partie d'un tout se met à fonctionner de façon autonome $e^{15}$, et comme y insiste Thomas Hunkeler, «[...] la logique du blason n'est pas celle du pars pro toto, mais celle du pars in loco totius : la partie choisie ne représente pas la dame, elle la remplace $»^{16}$. Le blason relève également de "la culture de la dissection $»^{17}$. En l'occurrence, cette a(na)tomisation fétichiste est une prise de possession de l'objet féminin par le sujet masculin. Comme l'explique Françoise Charpentier, il s'agit d'une " énumération où le nom épuise la chose et en donne la maitrise ». Cette critique parle encore de "corps aliéné » compte tenu de l'impossibilité du blason à faire « surgir un sujet vivant, l'image totalisante d'une personne $»^{18}$. C'est le blason synthétique qui domine chez les sonnettistes. Si l'on trouve des blasons des cheveux, de l'œil, du sein chez Ronsard, et du pied chez Du Bellay, leurs œuvres comptent davantage de blasons du corps.

La plupart des recueils de sonnets élisabéthains contiennent au moins un sonnet-blason relevant du premier type défini ci-dessus, le blason synthétique. Si ce type domine dans la production des poètes anglais, c'est peut-être parce que la tradition marotique leur est parvenue filtrée par les membres de la Pléiade. Ce sont surtout Sidney (cf. le sonnet 9 d'Astrophil and Stella, par exemple), Spenser (cf. les sonnets 15 et 64 d'Amoretti) et Shakespeare qui se distinguent en faisant preuve d'une bien plus grande liberté que les sonnettistes élisabéthains généralement considérés comme mineurs, entre autres parce que leur dépendance à l'égard de leurs sources est trop étroite ${ }^{19}$. Sous la plume de ces derniers, le sonnet-blason se réduit souvent à un inventaire des charmes

14. Pascal Quignard, Petits traités VII (Paris : Maeght, 1990), p. 123-24.

15. Cathy Yandell, «Iconography and Iconoclasm : The Female Breast in French Renaissance Culture », The French Review 83.3 (2010), p. 548.

16. Thomas Hunkeler, «Cadavres exquis. Du corps de la dame à la corporation des blasonneurs anatomiques ", in Arts littéraires, arts cliniques, éd. R. Fonkoua, B. Galtier et C. Jacot Grapa (Cergy-Pontoise : Université de Cergy-Pontoise : 2003), p. 81.

17. Cf. Jonathan Sawday, The Body Emblazoned. Dissection and the Human Body in Renaissance Culture (London, New York : Routledge, 1996), p. 196.

18. Introduction aux Euvres poétiques de Louise Labé et Pernette du Guillet (Paris : Gallimard, 1983), p. 14-15. Sur la poétique de la fragmentation déjà à l'œuvre chez Pétrarque, voir Nancy Vickers, « Diana Described : Scattered Woman and Scattered Rhyme », Critical Inquiry 8 (1981), p. 265-79.

19. Voir Janet Scott, Les Sonnets élisabéthains. Les sources et l’apport personnel (Paris : Champion, 1929). 
de la belle cruelle. La plupart des blasonneurs ne proposent que des variantes d'un même catalogue d'images si bien qu'il est possible de créer un portrait composite d'une Dame pétrarquiste à partir de simples extraits de sonnets de provenance diverse :

Combing her locks, of which the yellow gold

Made blush the beauties of (the Morn's) curlèd wire

(Thomas Lodge, Phillis 9, v. 6-7)

Her eyes the brightest stars the heavens hold

(Batholomew Griffin, Fidessa 39, v. 3)

Thy dainty cheeks, pure damask Rose breed

(Robert Tofte, Laura II, 16, v. 8)

Thy corall coloured lips

(anon., Zepheria, canzone 23, v. 1)

My smelling with her breath like Civet sweet

(Thomas Watson, Hekatompathia 54, v. 5)

in thy breath, that heavenly musick wons,

which whe[n] ye speak'st, Angels their voyces straines

(Henry Constable, Diana, $7^{\mathrm{e}}$ décade, 1 , v. 11-12)

the beauty of her Breasts [...]

This Venus' Bower, you rightly may compare

To whitest snow that e'er from heaven fell

(Laura I, 29, v. 5, 7-8)

In earth a goddess, what can be more strange ?

(Giles Fletcher, Licia 24, v. 12)

Lorsqu'il compose le sonnet 130, Shakespeare puise dans ce répertoire puisqu' il associe l'œil au soleil, la lèvre au corail, le sein à la neige, la chevelure à des fils d'or, les joues à des roses, le souffle à du parfum, la voix à une musique, et 
compare en outre la démarche de la Dame avec celle d'une déesse. Comme les sonnettistes-blasonneurs, il détaille les différentes parties du corps de la Dame. Cela dit, il est loin de respecter l'ordre de l'effictio tel que l'a prescrit Vinsauf : les yeux viennent avant les cheveux, les joues, après les lèvres et les seins, etc., ce qui constitue une première prise de distance à l'égard de son modèle. D'autre part, au lieu de reprendre à son compte les métaphores convenues, il ne les évoque que pour s'en désolidariser : tout son poème est construit sur le principe de négation. Dans ce sonnet, il prend le contre-pied de l'éloge pétrarquiste tel que le pratiquaient ses contemporains.

Plusieurs critiques estiment que Shakespeare, en écrivant ce texte, avait une cible bien précise, mais tous ne s'accordent pas sur la cible en question. Patrick Cruttwell constate que la plupart des images présentes dans le sonnet se trouvent dans le $7^{\mathrm{e}}$ poème de l'Hekatompathia (1582) de Thomas Watson ${ }^{20}$, blason canonique qui respecte les principes de l'effictio. Il contient sept éléments qui ont peut-être retenu l'attention de Shakespeare - la description des cheveux ("Her yellow locks exceed the beaten gold»), des yeux (" Her sparkling eyes in heav'n a place deserve »), des paroles ( Her words are music all of silver sound »), des joues (" On either cheek a Rose and Lily lies »), du souffle ( Her breath is sweet perfume, or holy flame»), des lèvres (« Her lips more red than any Coral stone ») et des pieds («Her slipper such as Momus dare not mock ») trouve un écho dans le sonnet 130. Cependant, comme le fait observer E. G. Rogers, si l'on admet que les sonnets à la Dame brune ont été conçus en 1598-1599, il est peu probable qu'un poème rédigé seize ans auparavant ait fait l'objet d'un traitement satirique - la satire aurait perdu de son mordant -, ce qui amène ce commentateur à proposer un hypotexte plus tardif, la Diella (1596) de Richard Linche, dont plusieurs passages ont pu suggérer des parallèles à Shakespeare ${ }^{21}$. Le début du sonnet 32 , avec son évocation des lèvres corallines, des yeux solaires, du souffle qui embaume et des joues de rose pourrait, en effet, être l'un des textes qu'il tourne en dérision.

Quant au $3^{\mathrm{e}}$ sonnet de Diella ${ }^{22}$, il contient la métaphore de la neige pour qualifier la blancheur de la peau, le terme « wire » qui désigne les cheveux 20. Patrick Cruttwell, The Shakespearean Moment and its Place in the Poetry of the $17^{\text {th }}$ Century (London : Chatto and Windus, 1970), p. 18-19.

21. E. G. Rogers, "Sonnet CXXX : Watson to Linche to Shakespeare ", Shakespeare Quarterly 11.2 (1960), p. 232-33.

22. Cf. Colin Burrow, Complete Sonnets and Poems (Oxford : Oxford University Press, 2002), p. 640. 
tressés en un fin réseau, et l'adynaton par lequel les yeux, tout en étant des fontaines de cristal, jettent des feux dont l'éclat dépasse en splendeur celui du soleil au zénith - trois éléments qui ont pu inspirer les saillies shakespeariennes :

my mistres snow-white skinne doth much excell

the pure-soft woll arcadyan sheepe doe beare;

Her hayre exceedes gold forc'd in smallest wire,

in smaller threds then those Arachne spun;

her eyes are christall fountaines, yet dart fire

more glorious to behold then Mid-day sun [...]

(v. 3-8)

Enfin, voici le $8^{\mathrm{e}}$ sonnet de Phillis (1593) de Thomas Lodge, parfois présenté comme le contre-modèle de l'auteur du sonnet $130^{23}$ :

No stars her eyes to clear the wandering night,

But shining suns of true divinity,

That make the soul conceive her perfect light !

No wanton beauties of humanity

Her pretty brows, but beams that clear the sight

Of him that seeks the true philosophy!

No coral is her lip, no rose her fair,

But even that crimson that adorns the sun.

No nymph is she, but mistress of the air,

By whom my glories are but new begun.

But when I touch and taste as others do,

I then shall write and you shall wonder too..$^{24}$

Les deux poèmes ont certains points communs : la structure du texte de Lodge est, elle aussi, entièrement négative comme l'atteste l'anaphore de no (vers 1,4 , 7,9); certaines métaphores convenues sont dénoncées - les yeux de la Dame ne sont pas des étoiles, sa bouche n’a rien du corail, sa beauté n'est pas celle d'une

23. C'est notamment l'hypothèse de Sidney Lee, éditeur des Sonnets en 1907. Cité par H. E. Rollins dans Sonnets (Philadelphia, London : New Variorum, 1944), p. 334.

24. Martha Foote Crow, éd., The Project Gutenberg EBook of Elizabethan Sonnet Cycles by Thomas Lodge and Giles Fletcher (EBook \#18841, 1996), n. p. 
rose, elle n'a rien d'une nymphe... Cependant, cette rhétorique sert un propos inverse de celle que déploie Shakespeare, car la négation vient paradoxalement soutenir la convention qu'elle paraît contester en contribuant à la surenchère inscrite dans le modèle pétrarquiste ${ }^{25}$ : qu'importe que les yeux de la Dame ne soient pas des étoiles puisqu'ils sont de divins soleils; si ses lèvres ne rappellent pas le corail ni sa beauté, la rose, leur teinte cramoisie est celle du soleil même ; elle n'est pas une nymphe, certes, mais elle est la maitresse des airs.

Que Shakespeare ait formé ou non le dessein de parodier l'un des blasons précités, nous ne le saurons sans doute jamais car il avait l'embarras du choix. Ce que l'on peut affirmer, en revanche, c'est que le sonnet 130 fonctionne comme leur métatexte et qu'il instaure une relation critique du même ordre avec tous les poèmes de leur classe.

Il ne s'agit pas, bien entendu, de prétendre que l'anti-pétrarquisme est une spécificité shakespearienne ${ }^{26}$. Philip Sidney, baptisé par ses admirateurs «le Pétrarque anglais $~^{27}$, n'en expose pas moins ses doutes quant au pouvoir d'évocation des mots (cf. les sonnets 3, 35, 37 d'Astrophil and Stella, par exemple) et plus précisément de la rhétorique galante, comme c'est le cas dans le sonnet 6 , dont voici le premier quatrain :

Some Lovers speake, when they their Muses entertaine,

Of hopes begot by feare, of wot not what desires,

Of force of heav'nly beames infusing hellish paine,

Of living deaths, deare wounds, faire stormes, \& freesing fires.

[Certains amoureux parlent, s'adressant à laur Muse,

d'espoirs nés de frayeurs, d'on ne sait quels désirs,

de la puissance de rayons célestes qui infligent des douleurs infernales,

de morts vivantes, blessures chères, belles tempêtes et feux glacés $]^{28}$

25. Déjà, Vinsauf proposait, dans l'un de ses exemples de description de belle femme, une liste des amantes de Jupiter, dont aucune n'égalait en beauté celle qu'il louait dans son effictio. Les poètes pétrarquisants, dont Marot dans son blason, recourent systématiquement à ce procédé.

26. Pour une approche globale de l'anti-pétrarquisme, voir Heather Dubrow, Echoes of Desire (Ithaca, London : Cornell University Press, 1995).

27. Cf. Lee, Elizabethan Sonnets VI, « The Earliest Elizabethan Sonneteers - Sidney and Watson », p. 15. Bartleby.com 2012.

28. Philip Sidney, Astrophel et Stella, trad. M. Poirier (Paris : Aubier, 1962), p. 50-51. 
Les sentiments exprimés - le désir, la crainte, l'espoir - l'image des rayons célestes dont les yeux de la Dame transpercent le Poète et les oxymores du quatrième vers sont caractéristiques du style de Pétrarque et des pétrarquisants. La suite du texte mentionne d'autres ingrédients propres à ce style : allusions mythologiques, pipeau pastoral, douce plainte, larmes et soupirs. Sa conclusion, selon laquelle le Poète n'a nul besoin de recourir à une telle panoplie puisqu'il lui suffit de proférer d'une voix tremblante son amour pour Stella ne doit pas faire oublier, d'une part, que Sidney n'a pas dédaigné de recourir ailleurs aux ingrédients en question, et d'autre part, que le concetto qu'il développe s'inscrit lui aussi dans la tradition pétrarquiste, ce qu'atteste le sonnet programmatique des Amours de Diane de Desportes - " Je n'agrandiray point, riche d'inventions, / Vos beautez, vos dédains, ma foy, mes passions : / Il suffira qu'au vray mon crayon se rapporte $»^{29}-$, l'allégeance à la nature étant une nouvelle pose poétique.

Comme le rappelle Patrick Cruttwell à propos de la période élisabéthaine, à peine les flots de recueils de sonnets s'étaient-ils déversés que sonnets et son-

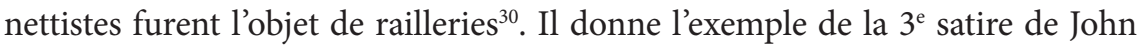
Marston, qui décrit l'amoureux transi murmurant un sonnet à sa belle dans un soupir, et dont la passion est si intense qu'il va jusqu'à couvrir ses fenêtres de semblables poèmes. Certains sonnettistes eux-mêmes, dont Sidney, Sir John Davies, et Shakespeare dans son théâtre ${ }^{31}$ se gaussaient de ce pétrarquisme outrancier, tendance dont on peut supputer qu'elle était d'autant plus marquée que ces auteurs arrivaient à la fin d'une tradition. En effet, plusieurs poètes élisabéthains manifestent une conscience aiguë de leur postériorité et semblent déplorer le fait de ne pouvoir s'affranchir du poids de leur(s) modèle(s). Dans le sonnet 35 de Delia, Samuel Daniel concède que sa Délie, bien qu'elle soit l'égale de Laure, ne saurait trouver en lui un Pétrarque - du moins peut-elle se consoler en se sachant aussi aimée. Dans le sonnet dédicatoire d'Ideas Mirrour (1594), Michael Drayton assure qu'il n'a imité servilement aucun de ses prédécesseurs, qu'il s'agisse de Pétrarque ou de Desportes, dénégation dont on peut inférer que l'imitation servile est la norme tant le modèle est pesant.

29. Philippe Desportes, Amours de Diane I, 1, v. 9-11 (Genève, Paris : Droz, Minard, 1959), p. 26.

30. Patrick Cruttwell, The English Sonnet (London : Longmans, Green and Co, 1966), p. 18.

31. Katherine Duncan-Jones relève que le dramaturge présente ses personnages amateurs de sonnets comme des imbéciles et tourne en dérision ceux qui composent des poèmes d'amour, des sonnets en particulier : K. Duncan-Jones, Shakespeare's Sonnets (London : Thomas Nelson and Sons, 1997), p. 46. 
Cela dit, pétrarquisme et anti-pétrarquisme coexistent déjà chez les poètes de la Pléiade. Dans l'« Élégie à son livre » qui ouvre le Second livre des amours (1560), Ronsard s'excuse d'être passé de l'éloge de Cassandre à celui de Marie : «Petrarque sur moy / N'avoit authorité de me donner sa loy $»^{32}$. Le carpe diem horatien l'emporte chez Ronsard sur le culte exclusif d'une Dame. Cependant, comme le notent les éditeurs du texte, «Il est rare qu'au XVI e siècle on s'en prenne à Pétrarque lui-même $»^{33}$. On voit plus fréquemment un auteur se distancier des conventions pétrarquistes qu'il a adoptées par ailleurs, comme Du Bellay, par exemple. Dans Divers Jeux rustiques (1557), le Poète peut parfaitement dire de sa Dame qu'« À l'or ses tresses font honte » et que «Sa main l'alebâtre passe $»^{34}$, proposer un catalogue en miniature des formules du maître toscan en évoquant les lèvres qui « Blêmissent le teint des roses ", « La neige des doigts polis, / Qui en dix perles finissent », «le blanc et vermeil / De cette face tant claire, / Dont l'un et l'autre soleil / À (ses) ténèbres éclaire », les ondes de ses « tresses blondelettes", " cette haleine tant douce », cette " voix céleste et non-pareille » ${ }^{35}$, et d'autre part, prétendre qu'il a « oublié l'art de Pétrarquiser » et rejeter " ce Paradis de belles fictions » dans un poème précisément intitulé "Contre les Pétrarquistes ». Pourtant, ce dernier texte énumère à plaisir les divers ingrédients qui composent le discours desdits poètes, dont il dénonce « Le parjure artifice » : ce ne sont qu' « or / Perles, cristal, marbre, et ivoire encor», "Fleurs, lis, œillets et roses ", "firmament [...] peint sur un beau front ", «beau teint de l'Aurore », « corps divin », « regrets et soupirs ». En outre, cet apparent renoncement à l'esthétique pétrarquisante s'achève par une abdication espiègle en faveur de son modèle, car si la Dame ne souhaite pas qu'il loue ses beautés "sans feindre ni pleurer ", il reviendra à son mode d'expression antérieur : «Si toutefois Pétrarque vous plaît mieux, / Je reprendrai mon chant mélodieux, / Et volerai jusqu'au séjour des Dieux / D’une aile mieux guidée $»^{36}$.

On retrouve dans le sonnet 130 de Shakespeare la pose qui consiste à se détourner des artifices mêmes dont on se délecte. Le Poète a beau écarter

32. Ronsard, Euvres complètes I, éd. Jean Céard et allii (Paris : Gallimard, 1993), p. 168.

33. Ronsard, Euvres complètes I, p. 1298.

34. Joachim Du Bellay, "De sa peine et des beautés de sa dame ", in Divers Jeux rustiques, éd. Ghislain Chaufour (Paris : Gallimard, 1996), p. 91.

35. Du Bellay, « Chant de l'Amour et du Printemps », Divers Jeux, p. 76-78.

36. Du Bellay, «Contre les Pétrarquistes », Divers Jeux, p. 103-10. 
l'association entre œil et soleil, lèvres et corail, sein et neige, etc., en soulignant l'inadéquation de ces rapprochements, il ne les réactive pas moins. Son texte se démarque d'une tradition dans laquelle, en même temps, il s'inscrit. Pour caractériser plus précisément son geste, disons qu'en inscrivant la tradition dans son poème, il montre qu'il l'a faite sienne au point de pouvoir en jouer avec une grande dextérité. Comme chez Du Bellay, on trouve chez Shakespeare un rapport ambigu au modèle, fait d'adhésion et de distance ludique. Cela dit, contrairement au poète de la Pléiade, l'auteur du sonnet 130 ne se propose nullement d'abdiquer en faveur de Pétrarque : il persiste dans l'anti-pétrarquisme en dénonçant les "fausses métaphores" (false compare) dont sont parées les Dames sous la plume des blasonneurs.

Faut-il considérer pour autant que Shakespeare a sacrifié à la mode inverse, celle du contreblason ? Ce dernier genre consistait à parodier le blason en évoquant non plus la splendeur du corps féminin, mais sa hideur. Là encore, Marot fut l'initiateur : à peine avait-il lancé le blason qu'il se mit à composer un contreblason ${ }^{37}$, réplique à son texte inaugural intitulée "Le Blason du laid tetin » (1536), dont voici l'incipit: «Tetin qui n'as rien que la peau, / Tetin flat, Tetin de drappeau, / Grande tetine, longue tetasse, / Tetin, doy je dire bezasse ${ }^{38}$. Marot était-il mû par « un penchant presque infaillible pour la contradiction $»^{39}$ ? On peut voir aussi l'apparition du contreblason comme une réaction à la mode du blason - un genre, dès lors qu'il est trop pratiqué, appelle sa parodie -, et rappeler que cette réaction est conforme à la « conflictualité interne » propre au maniérisme ${ }^{40}$. D’autre part, comme le souligne Nancy Vickers, le fait que les blasonneurs aient présenté la même partie du corps sous un jour tantôt favorable, tantôt défavorable, indique que l'enjeu pour ceux qui prenaient part à cette joute poétique était avant tout de rivaliser d'habileté rhétorique ${ }^{41}$. Marot

37. Rappelons que dès 1175, outre son sens héraldique de "bouclier » ou d'" armoiries sur bouclier ", le terme « blason " pouvait renvoyer à un "propos louangeux ou diffamatoire » : A. J. Greimas et T. M. Keane, Dictionnaire du moyen français, La Renaissance (Paris : Larousse, 1992).

38. Blasons anatomiques du corps féminin, version numérisée du CÉSR, p. 66.

39. M.-M. Fontaine, "Blasons » in Dictionnaire des littératures de langue française, éd. Jean-Pierre de Beaumarchais et allii (Paris : Bordas, 1994), p. 286.

40. Dubois évoque la « rhétorique du conflit interne, axée sur la négation et la dénégation, l'antithèse et l'oxymore, les jeux du pour et du contre (blasons et contre-blasons, érotique et contr' ou ant'érotique) »: Le Maniérisme, p. 183.

41. Vickers, « Members Only », p. 12. 
invita derechef ses confrères à le suivre, mais ils furent moins nombreux à s'exécuter ${ }^{42}$.

Bien avant lui, certains poètes avaient évoqué la laideur d'une femme, laideur souvent associée à la vieillesse, comme c'est le cas dans la $8^{e}$ épode d'Horace, par exemple. On trouve également des portraits de femme disgracieuse chez les poètes du Moyen Âge. Ainsi, la poignante ballade de François Villon intitulée «Les Regrets de la Belle Heaumière » présente, en reprenant le motif latin du Ubi sunt, un tableau contrasté de la beauté féminine canonique de l'époque médiévale et de la déchéance physique inéluctable dont se plaignent celles dont les attraits leur conféraient jadis un pouvoir sur les hommes.

Si Marot, qui connaissait parfaitement Villon pour l'avoir édité (et pillé à l'occasion $)^{43}$, a subi son influence, c'est sur un mode bien différent qu'il s'exprime lorsqu'il évoque la laideur d'une femme : qu'il s'agisse du «Blason du laid tetin » ou de l'épigramme intitulée « À une laide » — par laquelle il fait savoir à sa maîtresse que son " diable de visage » est si répugnant que pour qu'une étreinte entre eux soit possible, il faudrait qu'elle vienne ne le trouver «que de nuyt $»^{44}-$, c'est la veine satirique qu'il exploite pour le plaisir d'écrire «à contre poil » ${ }^{45}$.

Nous savons que l'œuvre de Marot était connue des poètes élisabéthains ${ }^{46}$. Cela dit, que les blasons et contreblasons français aient ou non traversé la Manche à l'époque, l'Angleterre n'était pas en reste et avait, en matière de portrait de femme laide, sa propre tradition. On songe, bien entendu, à la hideuse vieille femme ( the loathly lady») du conte narré par Alys de Bath dans les Contes de Cantorbéry de Chaucer, mais cet exemple n'est pas unique. C'est l'outrance comique qui caractérise «A Description of his Ugly Lady » de Thomas Hoccleve, poète du début du XV siècle : sa malheureuse Dame laide est affublée

42. Peletier du Mans rédigea un Contreblason du cœur pour faire pendant à son Blason du cœur, et Jean Rus procéda de même pour le nez. Quant au moraliste Charles de la Hueterie, il s'empara du contreblason dans un tout autre esprit que celui de Marot, son objectif étant de " glorifier l'âme » en dénigrant le corps. Cf. Saunders, "La beauté que femme doibt avoir" ", p. 40.

43. Voir Euvres poétiques II, p. 1087 et I, p. XIX.

44. Euvres poétiques II, p. 350.

45. « par maniere de rire, / Il m’est venu voulenté de descrire / A contre poil ung Tetin [...] » précise-t-il dans l'épître «A ceulx, qui apres l'Epigramme du beau tetin en feirent d'aultres »: Euvres poétiques I, p. 338.

46. Euvres poétiques I, p. 750. 
d'un petit front mesquin, de joues flasques, molles comme de la glaise, de mâchoires proéminentes, d'un nez tel un toit en saillie grâce auquel elle est assurée qu'il ne pleuvra pas dans sa bouche, d'épaisses lèvres grises, d'un menton qui se dérobe ; pour comble de disgrâce, son corps a la rotondité d'une balle et son chant est aussi mélodieux que celui d'un perroquet ${ }^{47}$. Enfin, dans son Arcadia (1590), Sidney se livre à une redistribution cocasse des différents éléments de l'effictio dans sa description de la sotte Mopsa - ses joues sont rapprochées de l'opale et non pas des roses, ses yeux, des perles au lieu du soleil, ses lèvres du saphir plutôt que du corail, et ainsi de suite (I, 3, v. 9-14). Mis en valeur par son insertion dans un texte en prose, ce sonnet qui brouille les codes du blason suffit à indiquer que Mopsa n'est pas une beauté.

Cela dit, que l'on songe au topos anglais de l'ugly lady ou à la parodie du blason à la française, ce n'est pas cette veine-là que Shakespeare exploite dans son sonnet 130 : l'enjeu n'est pas de détailler les traits disharmonieux de la physionomie d'une femme. Certaines critiques, pourtant, estiment que le texte porte sur la Dame un jugement dépréciatif. Pour Katherine DuncanJones, auteure d'une précieuse édition des sonnets, la femme qu'il dépeint est caractérisée par son teint brouillé, son haleine nauséabonde et sa démarche pataude $^{48}$. Une telle lecture est pour le moins hâtive : si les joues de la Dame n'ont ni la teinte ni l'aspect soyeux des roses, faut-il en déduire que sa carnation n'est pas irréprochable - hypothèse qu'aucun élément du texte ne vient corroborer $^{49}-$ ou que la métaphore pétrarquiste consistant à rapprocher ses joues

47. R. T. Davies, éd., Medieval English Lyrics (London : Faber and Faber, 1963), p. 165.

48. Duncan-Jones, Shakespeare's Sonnets, p. 48.

49. "Shakespeare has made it abundantly clear...that the lady was no beauty " estime Brandes, auteur d'un William Shakespeare (1898), cité par H. E. Rollins, dont voici l'objection : « This idea, often repeated, is refuted by the final couplet. The "thousand errors" that the poet's eyes see in her (141.2) may, or may not, concern her beauty or lack of it ; but Sh. does not say here that his mistress's breasts are dun in color, her hair black wires, her breath bad, her voice unpleasing, her feet flat» (p. 331). Il existe un débat sur la signification du verbe reek au vers 8 (cf. Stephen Booth, Shakespeare's Sonnets [New Haven, London : Yale University Press, 1980], p. 454-55). Si le terme pouvait être pris en mauvaise part, y compris par Shakespeare dans des contextes guerriers en particulier (on songe aux " reeking wounds » de Macbeth, I. ii. 34), il pouvait aussi être neutre ou positif comme l'indique un exemple de 1542 : «perfume being poured... reeketh into the air " $(O E D)$. Shakespeare emploie le terme de façon ludique dans des contextes amoureux, pour évoquer la concupiscence de Vénus (Venus and Adonis, v. 555) ou se moquer des soupirs d'un galant (Love's Labour's Lost, IV. iii. 138). Il n'est donc guère possible de déduire de la présence du verbe reek dans le sonnet que l'image que ce dernier donne du corps féminin est négative. 
des roses n'est pas pertinente ? Comme Richard Halpern ${ }^{50}$, Thomas P. Roche ${ }^{51}$ et Frank Erik Pointner, dont le jugement a été cité précédemment, Katherine Duncan-Jones considère que c'est la Dame brune qui est mise en cause et lit donc le poème comme un contreblason de type marotique, ce qu'il n'est pas, me semble-t-il.

Pour le montrer, posons la question suivante : de quoi au juste le sonnet 130 est-il la parodie ? S'il peut être qualifié de contreblason, en quel sens faut-il l'entendre ? Dans ce poème, Shakespeare joue avec les attentes de son lectorat : les douze premiers vers peuvent faire croire que le Poète dénigre sa Dame en multipliant les négations et les comparaisons défavorables ${ }^{52}$. Si, de la Laure du Chansonnier à celle de Robert Tofte (Laura, 1597), la splendeur de toutes les Dames pétrarquistes surpasse celle du soleil, du corail ou de la rose, la Dame brune est loin de leur être comparable. Mais est-il souhaitable qu'elle le soit ? Telle est la question, à laquelle le Poète répond par la négative, mais ce n'est que dans les deux derniers vers que l'ambiguïté est dissipée, d'où la surprise que suscite la pointe. Ce sonnet maniériste exige donc une lecture rétrospective, la clef de l'interprétation se trouvant dans le distique.

Certains indices doivent pourtant nous faire tendre l'oreille dès le début du poème : lorsque le virtuose de la langue anglaise qu'est Shakespeare écrit un vers aussi malhabile que "Coral is far more red than her lips' red ", il y a lieu de s'interroger sur l'effet que produit la duplication de l'adjectif red dans le contexte. En l'occurrence, le sonnet 130 imite à des fins burlesques une caractéristique stylistique de la poésie pétrarquiste - du blason en particulier $^{53}$ - à

50. Richard Halpern, Shakespeare's Perfume. Sodomy and Sublimity in the Sonnets, Wilde, Freud, and Lacan (Philadelphia : University of Pennsylvania Press, 2002), p. 30 : « [...] Shakespeare's Dark Lady is [...] the subject of an anti-blazon ».

51. Thomas P. Roche, Petrarch and the English Sonnet Sequences (New York: AMS Press, 1989), p. 384 : "Shakespeare does have parody in mind not against the belying classical conventions but against his lady [...] ». Plus loin, Roche évoque « the anti-blazon of his mistress ».

52. "Shakespeare's mock-blazon has sometimes been thought misogynistic, in part because readers have formed their idea of it from its octave, where nothing positive is predicated of the mistress. (Of course, nothing negative is predicated of her either.) " note Helen Vendler dans The Art of Shakespeare's Sonnets (Massachusetts : The Belknap Press of Harvard University Press, 1997), p. 557.

53. "Anaphora is central among [the figures of repetition] and is perhaps the figure most characteristic of the genre » relève Vickers, dans « Members Only », p. 15. 
savoir la répétition sous toutes ses formes ${ }^{54}$ qui, bien qu'elle traduise l'obsession fascinée du Poète pour la Dame, n'en confère pas moins à bien des textes en question une pesante mécanicité ${ }^{55}$. Qu'il s'agisse de l'allitération (en /g/ dans grant, goddess, go, ground, v. 11-12, par exemple), de l'assonance (du phonème le/ dans red, then, breasts, hairs /eə/, head, v. 2-4), de la paronomase (white, why, wires, v. 3-5), de l'écho sonore (And in / Than in, v. 7-8), de la réitération de termes (red, v. 2 et 5 ; white, v. 3 et 5 ; wires, v. 4 ; roses, v. 5 et 6 ) ou du parallélisme de structure (If snow be white / If hairs be wires, v. 3-4; (far) more (than), v. 2,7 et 10 ; (and) yet, v. 9 et 13), le poète singe ses contemporains pour mieux s'en distinguer, comme l'indique assez la construction négative du sonnet.

C'est le fonctionnement de la poésie pétrarquiste que le sonnet 130 nous invite à examiner. Dans cette perspective, si le corail est bien plus rouge que celui des lèvres de la Dame, ce ne sont pas ces dernières qui sont imparfaites mais le corail en tant que terme comparant, et il en va de même pour tous les phores proposés (le soleil, la neige, les roses...). Au vers 3, par exemple, il ne s'agit pas de dire que les seins de la Dame sont d'un terne brun grisâtre (dun), mais que c'est l'aspect qu'ils auront si l'on retient la neige comme phore. Ce dernier est-il pertinent ? Ce n'est pas ce que suggère le why then, élément de distanciation que l'on peut traduire par « dans ces conditions » (conditions que l'on peut accepter ou non) et qui souligne le caractère arbitraire du rapport établi entre comparant et comparé. L'anaphore de la proposition conditionnelle (If snow be white / If hairs be wires, v. 3-4) renforce ce dernier trait.

L'expression If hairs be wires renvoie à un cliché poétique de la Renaissance qui prête aux cheveux des dames l'élégance de la passementerie : il faut sous-entendre golden wires, les fils d'or que l'on trouve dans l'Epithalame de Spenser (v. 154), dans le sonnet 17 de l'anonyme Zepheria ou encore dans l'incipit du sonnet 39 de la Fidessa de Griffin. La tradition pétrarquiste exalte la blondeur de la Dame idéale, or la Dame du sonnet 130 est brune, ce qui

54. Les blasonneurs y recourent si fréquemment que ce trait est parfois considéré comme inhérent au genre qui a leur prédilection. Saunders, The Sixteenth-Century Blason Poétique, p. 78.

55. Shakespeare souligne l'aspect mécanique de son premier quatrain en y introduisant deux vers monosyllabiques consécutifs (v. 3 et 4). Pour illustrer l'effet de pesanteur induit par la répétition, on peut songer au sonnet 17 de Coelia (1594) de William Percy, qui réitère l'impératif relent de façon obsessionnelle; le recueil contient en outre des exemples de l'épanalepse très prisée des Elisabéthains qui consiste à reprendre au début d'un poème le dernier vers du poème précédent, ce qui peut créer un effet de lassitude (17-19). 
constitue une transgression. Néanmoins, en conclure que le Poète émet une réserve quant à sa beauté serait aller vite en besogne : d'une part, dans d'autres sonnets de Shakespeare, l'association conventionnelle, que la langue anglaise souligne, entre blondeur et beauté - fair signifie à la fois «blond» ou «clair » et " beau »- est contestée ${ }^{56}$. D’autre part, des auteurs pourtant marqués par Pétrarque, qu'il s'agisse de Marot et de Ronsard en France ou de Sidney en Angleterre, n'ont pas hésité à chanter les louanges de leurs brunes beautés - Anne (d'Alençon), Marie (de Bourgueil) et Stella (Penelope Devereux) étaient toutes trois $"$ brunettes $\|^{57}$. Par-delà la liberté que prennent ces poètes à l'égard de la norme pétrarquiste, c'est le bien-fondé de la normativité en matière d'esthétique qu'ils interrogent.

Un autre détail suggère que les mérites de la Dame ne sauraient se mesurer à l'aune des conventions poétiques. Dans l'expression «I have seen roses damasked, red and white» (v. 5), le verbe damasked (damaskt dans l'in-quarto de 1609, édition de référence) est un hapax, une forme dont il n'existe qu'un exemple dans le corpus shakespearien ${ }^{58}$. Dès lors, il convient de se poser la question suivante : pourquoi Shakespeare n'a-t-il pas écrit "I have seen damask roses, red and white ", damask ayant un statut adjectival, conformément à son habitude ${ }^{59}$ et à celle de ses contemporains ${ }^{60}$ ? Ce ne sont pas des raisons

56. Voir les sonnets 127 et 131.

57. En outre, la Bible proposait un précédent avec la fameuse déclaration du cantique des cantiques ( 1 , 5), « Je suis noire, mais je suis belle » — la conjonction 'mais' indique assez qu'aux temps bibliques déjà, la blondeur était valorisée et qu’une beauté brune constituait un écart par rapport à la norme. Bettella montre que la célébration de la beauté brune était fréquente dans la poésie italienne à la fin du XVI siècle et au XVII ${ }^{e}$ siècle et qu'elle a même donné lieu à une mode dans les cercles littéraires vénitiens autour des années 1620 : Patrizia Bettella, The Ugly Woman. Transgressive Aesthetic Models in Italian Poetry from the Middle Ages to the Baroque (Toronto, Buffalo, London : University of Toronto Press, 2005), p. 133-52.

58. W. G. Ingram et Theodore Redpath précisent dans Shakespeare’s Sonnets (London : University of London Press, 1964), p. 298 : "This is the only use in Shakespeare of "damask" as a verb. 'Damask' occurs four times in Shakespeare as an attribute adjective and twice as an adjectival noun ».

59. Cf. « damask cheek» (Twelfth Night, II. iv. 112) et "Gloves as sweet as damask roses » (The Winter's Tale, IV. iv. 219).

60. Ingram et Redpath citent la $16^{\mathrm{e}}$ ode du Parthenophil and Parthenophe (1593) de Barnabe Barnes : «Her cheeks to damask roses sweet / In scent and colour were so like [...]» (Shakespeare's Sonnets, p. 300). Dans la même œuvre, j'ai également trouvé « sweet damask rosebud » et « O damask rose » (46, v. 6 et 14). Dans Laura, Tofte emploie le terme trois fois : « Thy dainty cheeks, pure damask Rose breed » (II, 16, v. 8) ; « Rich 
d'ordre métrique qui expliquent son choix puisqu'il s'agit, dans les deux cas, de pentamètres. L'explication est donc ailleurs. Employé comme adjectif, damask confère aux joues des Dames pétrarquistes les propriétés des roses de Damas, leur couleur diaprée, leur toucher velouté, et peut-être leur fragrance ; en tant que verbe, par contre, le terme implique l'intervention d'un agent - il ne s'agit plus d'évoquer simplement les roses de Damas, mais des roses que l'on aurait "damassées », auxquelles on aurait imposé un processus de dénaturation. Peuton considérer que cette modification que l'on fait subir à l'œuvre de la nature suggère, par analogie, l'artifice dont usent les poètes élisabéthains lorsqu'ils comparent les joues de leur Dame aux roses de Damas, les deux opérations faisant l'objet d'une réprobation implicite ? Telle est du moins mon hypothèse : grâce à l'ajout d'une consonne, l'adjectif devenu verbe, mis en relief au centre du vers, permet à Shakespeare de se démarquer de l'expression convenue tout en attirant l'attention sur elle. En somme, le phonème / $t /$ constitue l'indice d'une insurrection du texte shakespearien contre la métaphore trop attendue qu'est damask cheeks ( joues vermeilles») ${ }^{61}$.

Le poème tout entier s'appuie sur la littéralisation de la métaphore pour mieux faire apparaître la disjonction entre comparant et comparé et contester la pertinence du lien qui les unit : «dans la nature, les choses ne sont pas ainsi » semble-t-il nous dire. Il procède par désidéalisation, mais ce n’est pas au détriment du personnage féminin que celle-ci s'effectue : c'est une dénudation facétieuse des modes représentatifs conventionnels qu'il opère. Ainsi, l'expression «I grant I never saw a goddess go » (v. 11) fait songer à la répartie qu’un poète narquois pourrait adresser à l'un de ses confrères pétrarquistes trop enclin à prêter à sa Dame les attributs de la divinité. Giles Fletcher, qui est friand du verbe grant - je l'ai relevé onze fois dans Licia (1593) - et fait défiler dans ses poèmes un long cortège de déesses ${ }^{62}$, pourrait être ce confrère. Helen Vendler relève à juste titre l'humour des vers 11 et 12 , par lesquels le Poète fait mine

Damask Roses in fair cheeks do bide... » (II, 38, v. 1) ; « Wherewith she blushed, in show like damask rose » (III, 28, v. 6). Dans The Legend of Cassandra (1595) de Richard Barnfield, un amant embrasse sa bien-aimée et l'appelle « wanton Venus milk-white Dove, / Whose ruddie lips the damaske roses decke » (v. 10). Dans toutes ces citations, damask est un adjectif qui précède immédiatement rose ou rosebud.

61. Je remercie la linguiste Patricia Buccellato de m'avoir confirmé la validité de cette analyse.

62. Mentionnons deux poèmes de Licia dans lesquels, coïncidence troublante, les termes " grant " et " goddess " sont utilisés au sein d'un même vers : "I grant a goddess, but not half do mild » (11, v. 10) et « The heavens did grant; a goddess she was made» $(24$, v. 9$)$. 
de croire qu'il n'appartient pas à la caste de privilégiés qui ont eu le bonheur d'apercevoir une déesse ${ }^{63}$. La naïveté feinte qui consiste à prendre à la lettre une succession de métaphores pour mieux en souligner l'artifice atteint son paroxysme à la fin du dernier quatrain.

Compte tenu du ton enjoué du sonnet, il est difficile de souscrire à la lecture de Paul R. Thomas selon laquelle l'amant remercie les puissances célestes («by heaven », v. 13) d'avoir une telle maîtresse en un temps où les femmes en chair et en os ne sont ni blanches comme neige, ni belles comme les roses... ${ }^{64}$. Je discerne, pour ma part, un emploi ludique de l'expression by heaven qui accentue le mouvement anti-pétrarquiste et anti-néoplatonicien du texte - loin du Ciel qu'invoque Pétrarque ${ }^{65}$, le ciel, en l'occurrence, est réduit à une interjection, dont le Poète use non sans malice pour célébrer, non plus une déesse, mais une beauté toute charnelle. Fidèle à l'esprit de sérieux qui caractérise son approche du texte, Paul R. Thomas considère encore que le sonnet est un éloge de la femme moderne, avec tous ses défauts ${ }^{66}:$ l'illusion référentielle semble fonctionner trop bien pour ce critique - la Dame que Shakespeare construit est un être de fiction, l'allégeance implicite du texte à la nature étant, comme chez Desportes et Sidney, une nouvelle pose poétique. On peut situer Stephen Booth à l'autre extrémité de l'axe interprétatif : à en croire ce lecteur pourtant perspicace et érudit de la poésie de Shakespeare, le texte est une séduisante bagatelle qui n'a d'autre but que celui d'amuser ${ }^{67}$. Un tel commentaire parait réducteur car si le sonnet 130 est indéniablement un grand poème comique où l'on ne détecte aucun didactisme empesé, c'est aussi une leçon de lecture qu'il nous offre : en tendant un miroir au blason, l'œuvre devient une méditation sur le genre littéraire qu'elle reflète ${ }^{68}$.

63. Vendler, p. 557.

64. Paul R. Thomas, "Shakespeare's Sonnet 130 and the History of Two Ideas : The Effictio and the Topos of the World Upsidedown ", Encyclia 66 (1989), p. 78.

65. Dans le Chansonnier, le Poète prend Amour à témoin : Laure est «sur terre l'éclat qui nous fait voir le ciel » (192, v. 4). En dépit de la sensualité des textes, l'union à laquelle aspire le Poète n'a rien de profane : « [...] je chante sa divine beauté, / afin que de ma chair quand serai libéré / le monde sache bien comme ma mort est douce » $(217$, v. 112-14).

66. «This sonnet praises the modern woman, warts and all [...]». Thomas, p. 78

67. Booth, p. 452-54.

68. "Art develops via reflection of and on preceding art, where "reflection" signifies both a "mirroring" [...] and a "re-thinking" " [ "L'art évolue par le biais de la réflexion de et sur les ouvres antérieures, 
Pour mieux saisir le geste shakespearien, confrontons le sonnet 130 à d'autres poèmes de la Renaissance. Opposons-le tout d'abord au « Contreblason du tetin " par lequel Charles de la Hueterie brocarde le blason de Marot en des termes qui rappellent les vers 5 et 6 du poème de Shakespeare :

E le Tetin demeurera il la,

Ce fut Clement qui premier en parla :

J'ay veu cerise au soir \& au matin,

Oncq n'en vey croistre dessus tetin. ${ }^{69}$

En effet, les cerises n'ornent pas plus la poitrine des femmes que les roses ne fleurissent sur leurs joues. Cela dit, la mise en question du cliché poétique est le seul point commun aux deux textes car la satire de La Hueterie porte essentiellement sur le corps féminin comme l'indique la suite du poème, son distique final en particulier : "Sur estomach ce sont deux grosses louppes / Lavées au soir \& couvertes d'estouppes ».

Voici, à présent, les quatrains du sonnet 91 des Regrets Du Bellay :

O beaux cheveux d'argent mignonnement retors !

O front crêpe et serein ! et vous, face dorée !

O beaux yeux de cristal ! ô grande bouche honorée,

Qui d'un large repli retrousses tes deux bords !

O belles dents d'ébène ! ô précieux trésors,

Qui faites d'un seul ris toute âme énamourée !

O gorge damasquine en cent plis figurée!

Et vous, beaux grands tetins, dignes d'un si beau corps $!^{70}$

Il s'agit d'un éloge parodique ${ }^{71}$, d'un contreblason de type marotique dont l'habileté consiste à conserver les traits de la rhétorique galante (l'inter-

\footnotetext{
"réflexion" étant entendu au double sens de "reflet en miroir" [...] et de "re-considération"] dit George Steiner dans Real Presences (London : Faber and Faber, 1989), p. 17. (Ma traduction.)

69. Blasons anatomiques du corps féminin, Version numérisée du CÉSR, p. 67.

70. S. de Sacy, éd., Les Regrets. Les Antiquités de Rome (Paris : Gallimard, 1967), p. 129.

71. Le prototype de cet éloge parodique semble être un sonnet de Berni, "Chiome d'argento ", (in Patrizia Bettella, The Ugly Woman, p. 115-16). Voici ma traduction : "Cheveux d'argent fin, hirsutes
} 
jection « ô » qui traduit l'admiration ${ }^{72}$, les adjectifs laudatifs comme « beaux ", " serein ", " dorée ", " honorée », " précieux »..., les formulations hyperboliques - «Qui faites d'un seul ris toute âme énamourée ! ) tout en inversant les paramètres de la beauté pétrarquiste : les cheveux ne sont plus d'or mais d'argent, ce qui suggère la vieillesse ; au lieu d'être finement ourlée, la bouche est démesurée; l'ébène se substitue à l'ivoire ou aux perles pour qualifier les dents, que l'on imagine gâtées ; la gorge de marbre ou d'albâtre est à présent toute plissée par les ans; les seins sont devenus volumineux; et l'on découvre dans les tercets que la main a perdu sa finesse et sa longueur, les ongles, leur blancheur, les cuisses, leur rondeur, et les jambes, leur galbe. Ainsi vidé de son contenu, le discours amoureux devient risible. Comme Shakespeare, Du Bellay s'en prend à la convention en la parodiant, mais contrairement au poète anglais, il se moque à la fois de la Dame et des lieux communs.

De la même façon, Étienne Jodelle allie dénigrement du corps féminin et mise en cause de la tradition pétrarquiste dans le dernier sonnet des Contr'amours (1574) :

Combien de fois mes vers ont-il doré

Ces cheveux noirs dignes d'une Meduse?

Combien de fois ce teint noir qui m’amuse,

Ay-je de lis et roses coloré?

et entortillés / maladroitement autour d'un beau visage d'or ; / front plissé, que je ne peux regarder sans pâlir, / où l'Amour et la Mort brisent leurs traits. / Beaux yeux de perle, rayons qui se détournent / de toute chose qui leur est dissemblable ; / cils de neige et, ce qui m'afflige profondément, / cette main, ces doigts délicieusement épais et courts. / Lèvres de lait, grande bouche céleste ; dents d'ébène, rares et vagabondes ; / harmonie inouïe, ineffable ; / manières hautaines et solennelles ; à vous, divins / serviteurs de l'Amour, / j'atteste que telles / sont les beautés de ma dame ». Bettella rapproche le sonnet 130 d'un madrigal de l'Arétin, éloge parodique qu'un soupirant cynique adresse, pour se moquer d'elle, à une femme aux mœurs légères qui se pique de littérature sans rien y connaitre (p. 121-22). Bettella n'exclut pas la possibilité que Shakespeare ait eu connaissance de ce madrigal, publié à Londres en 1584, dont elle relève la proximité avec le sonnet 130 tout en soulignant sa différence : les deux textes ont une structure négative et ont pour effet de briser le stéréotype qui consiste à présenter la Dame pétrarquiste sous les traits d'une déesse, mais tandis que le madrigal s'achève sur une évocation répugnante, le sonnet déconstruit avec humour l'image divine de la Dame dont il propose néanmoins une description positive (p. 221, 122).

72. Du Bellay lui-même y a recouru, dans le sonnet 58 de L'Olive, par exemple : «O doulx pleurer ! ô doulx soupirs cuisans ! / O doulce ardeur de deux Soleilz luisans ! » (p. 23). 
Combien ce front de rides labouré

Ay-je applani ? et quel a fait ma Muse

Ce gros sourcil, où folle elle s'abuse,

Ayant sur luy l'arc d'Amour figuré ?

Quel ay-je fait son œil se renfonçant?

Quel ay-je fait son grand nez rougissant?

Quel ay-je fait sa bouche, et ses noires dents quelles?

Quel ay-je fait le reste de ce corps?

Qui, me sentant endurer mille morts,

Vivoit heureux de mes peines mortelles. ${ }^{73}$

Jodelle va plus loin que Du Bellay dans la dénudation des procédés littéraires: dans ce poème, la transformation que le poète fait subir à sa «Muse » pour en faire une Dame est explicite. Jamais le false compare dont parle Shakespeare dans le sonnet 130 n'aura été plus clairement illustré. Mais là s'arrête l'analogie entre les deux textes. En l'occurrence, c'est l'auto-flagellation que pratique le Poète dans la mesure où il regrette de s'être prêté à un jeu peu glorieux : c'est en pure perte qu'il s'est évertué à embellir une ingrate.

Tout autre est le ton du sardonique Drayton, dont voici le sonnet 8 d'Idea (1619) :

There's nothing grieves me, but that Age should haste,

That in my dayes I may not see thee old,

That where those two cleare sparkling Eyes are plac'd,

Only two Loope-holes, then I might behold.

That lovely, arched, yvory, pollish'd Brow,

Defac'd with Wrinkles, that I might but see ;

Thy daintie Hayre, so curl'd, and crisped now,

Like grizzled Mosse upon some aged Tree;

Thy Cheeke, now flush with Roses, sunke, and leane,

Thy Lips, with age, as any Wafer thinne,

Thy Pearly Teeth, out of thy Head so cleane,

That when thou feed'st, thy Nose shall touch thy Chinne :

These Lines that now thou scorn'st, which should delight thee,

Then would I make thee read, but to despight thee.

73. E. Buron, éd., Les Amours (Saint-Étienne : Publications de l'Université de Saint-Étienne, 2003), p. 165. 
[La seule chose qui m'afflige, c'est que la vieillesse viendra, et que de ma vie je ne te verrai pas vieille. À l'endroit où se trouvent ces deux clairs yeux étincelants, je n'apercevrai alors que deux trous. Ah, que je puisse seulement voir ce beau front bombé d'ivoire poli défiguré par les rides! Tes beaux cheveux à présent si bouclés et frisés devenus semblables à la mousse grisonnante sur quelque vieil arbre ; ta joue, or empourprée par les roses, enfoncée et maigre, tes lèvres amincies par l'âge semblables à une gaufrette ; tes dents de perle toutes tombées de ta tête, de sorte que lorsque tu manges ton nez touche ton menton! Ces vers qui devraient te réjouir, et qu'à présent tu dédaignes, je te les ferais lire alors pour te mortifier ! $]^{74}$

Ce n'est pas contre lui-même mais uniquement contre la Dame que le Poète décoche ses flèches. Ce sonnet, dont l'outrance rappelle la description que fait Hoccleve de son "ugly lady ", rompt avec le modèle inauguré par Pétrarque, dans la mesure où il superpose le portrait d'une beauté idéale et celui d'une femme défigurée par l'âge. Cela dit, contrairement aux poèmes de Du Bellay et de Jodelle, cet écart par rapport au modèle ne s'accompagne d'aucune mise en question des clichés de la poésie pétrarquiste que d'ailleurs il reprend à son compte : l'éclat solaire des yeux, l'ivoire du front, les roses qui donnent leur teinte aux joues et les perles qui ornent la bouche de la Dame sont autant de métaphores qui demeurent intactes dans le texte. Ce contreblason dans lequel le Poète se montre aussi cruel que la Dame se situe aux antipodes du poème 30 de la Delia de Daniel, qui traite du même thème : dans ce sonnet, le Poète compatissant imagine que ses vers sauront consoler la Dame en proie à la détresse lorsqu'elle constatera l'outrage des ans : "Goe you my verse, goe tell her what she was; / For what she was she best shall finde in you " [ "Allez, mes vers, allez lui dire ce qu'elle fut, / Car ce qu'elle fut, c'est en vous qu'elle le découvrira le mieux $»]^{75}$.

74. W. Hebel, éd., The Works of Michael Drayton, vol. II (Oxford : Blackwell and Mott, 1961), p. 314. Traduction Scott, Les Sonnets élisabéthains, p. 121.

75. (Ma traduction.) Le thème de la beauté déchue est fréquent à la Renaissance : outre Berni, Du Bellay et Jodelle, il a été traité par Le Tasse, Desportes, Ronsard et Donne. Si Daniel s'inspire de Desportes (cf. Scott, p. 120), toute idée de vengeance disparaît chez lui. On retrouve dans le célèbre sonnet 43 du Second Livre des Sonnets pour Hélène l'idée que la beauté de la Dame est contenue dans les vers du poète et qu'elle s'émerveillera en les répétant quand elle sera «bien vieille, au soir à la chandelle ", mais Ronsard, comme à l'accoutumée, conclut par le motif du carpe diem (p. 400-01). Quant au poème de Drayton, son ton grinçant rappelle Berni. Bettella considère que l'éloge paradoxal de la femme laide dont ce dernier a donné un exemple célèbre est une occasion manquée de mettre en question la 
S'il est légitime d'établir des analogies partielles entre certains des poèmes précités et le sonnet 130 de Shakespeare, ce dernier s'en distingue par un aspect déterminant : la nature du regard posé sur la Dame. Le texte shakespearien est bien plus proche du sonnet 18 de la Chloris (1596) de William Smith ${ }^{76}$, à mes yeux le plus réussi d'un recueil qui suscite habituellement des commentaires peu amènes ${ }^{77}$, et dont le seul sous-titre est de mauvais augure - The Complaint of the Passionate Despised Shepheard.

My Love, I cannot thy rare beauties place.

Under those formes which many writers use,

Some like to stones compare their mistris face

Some in the name of flowers do love abuse :

Some makes their love a goldsmiths shop to be,

Where orient pearles and pretious stones abounde.

In my conceite these farre do disagree,

The perfect praise of beautie foorth to sounde.

O Chloris thou dost imitate thy selfe,

Selfs imitating passeth pretious stones,

Or all the Easterne Indian golden pelfe :

Thy red and white with purest faire attones,

Matchlesse for beautie nature hath thee framed,

Onely unkinde and cruell art thou named.

Sidney Lee classe William Smith parmi les «poetæ minimi » et le présente comme un humble disciple de Spenser ${ }^{78}$. Pourtant, à en juger d'après ce poème qui semble railler les blasons floral (v. 4) et gemmologique ${ }^{79}$ (v. 5-6) de son prétendu maitre, ce n'est pas la déférence qui caractérise l'auteur, quelle que

représentation des femmes en littérature (p. 115), commentaire également applicable à Drayton et à bien des contreblasonneurs.

76. Il convient de mentionner aussi le sonnet 47 de Chloris, texte malheureusement tronqué, dont la structure négative semble servir le même propos que le sonnet 130.

77. Voir Scott, p. 200, et Lu Emily Pearson, Elizabethan Love Conventions (London : George Allen and Unwin, 1933), p. 124. Quant à J. W. Lever, il ne mentionne pas seulement le nom de William Smith dans un ouvrage pourtant intitulé The Elizabethan Love Sonnet (London : Methuen, 1966).

78. Elizabethan Sonnets X, « Poetæ Minimi », p. 6. Voir note 27.

79. Cf. les sonnets 64 et 15 d'Amoretti. 
soit l'étroitesse de sa palette. Le sens de la structure n'est pas son point fort : il revient aux pierres précieuses à deux reprises (v. 5-6, v. 10) après les avoir mentionnées une première fois (v. 3) pour se tourner ensuite vers les fleurs. L'unité de ton n'est pas non plus ce qui définit le mieux son art : le savoureux persiflage des vers 5 et 6 est suivi d'une réflexion empreinte de gravité, avant que le poème ne s'achève sur une platitude. En effet, alors que tout le texte opère un détachement à l'égard de la rhétorique pétrarquiste, le distique final renoue avec la convention en évoquant la " belle et cruelle Dame ${ }^{80}$ chantée par tous les sonnettistes.

Cela dit, le refus des comparaisons stéréotypées («I cannot», v. 1), la mise en cause des pratiques de ses contemporains («those formes which many writers use », v. 2 ; « compare», v. 3 ; « abuse», v. 4), le constat de l'inadéquation des outils poétiques destinés à l'éloge de la beauté (v. 7-8), le souci de vérité (« thou dost imitate thyself », v. 9) et la référence à la nature (v. 13) sont autant d'éléments qui permettent de rapprocher ce texte du sonnet 130 de Shakespeare. Si ce dernier est infiniment plus subtil, ne serait-ce que parce que tous ces griefs y sont implicites et traités sur le mode ludique, les deux poèmes apportent la preuve qu'un sonnet peut être anti-pétrarquiste sans être un contreblason marotique : contrairement aux œuvres précitées de La Hueterie, Du Bellay, Jodelle et Drayton, ce n'est pas la beauté de la Dame qui y est mise en question, ce sont les modalités de sa représentation.

Sous la plume de Shakespeare, le contreblason n'est plus le revers d'un blason montrant la Dame sous un jour défavorable, c'est un blason sur le blason qui s'adresse à un lectorat averti, à un public de connaisseurs au fait des codes poétiques qui prévalent en son temps. Quiconque n'aurait jamais lu de sonnet-blason serait bien en peine de saisir la portée du texte : nous l'avons vu, un décodage générique s'impose.

On peut supputer que les critiques qui interprètent le texte comme un contreblason marotique se laissent influencer par ce qu'ils ou elles savent des autres poèmes relatifs à la Dame brune - les sonnets 129, 141 et 144, en particulier -, ou plus exactement par la lecture qu'ils ou elles en font. DuncanJones va jusqu'à déplorer «la scandaleuse misogynie » des sonnets 127 à $154^{81}$,

80. J'emprunte la formule aux Amours de Diane de Desportes (I, 13).

81. Duncan-Jones, p. 50. Employé globalement, le terme de "misogynie » paraît discutable dans la mesure où même dans les textes les plus hostiles à la Dame brune comme le sonnet 141, qui esquisse un contreblason marotique, la Dame n'est pas attaquée dans sa féminité mais en tant qu'objet de désir — le 
prêtant ainsi à cette partie du recueil une stabilité qu'elle est loin d'avoir. En effet, aucune description homogène de la Dame n'y est proposée ${ }^{82}$ : plutôt qu'un

sujet du poème est moins la Dame elle-même que la division interne que subit le Poète en proie à un désir qui l'aliène. De la même façon, le sonnet 129 contient moins une réprobation de la Dame que de la luxure, même si c'est la première qui suscite la seconde. Enfin, le sonnet 144 maudit la Dame, non pas en tant que femme mais en tant qu'agente d'une triangulation intempestive du désir. On rencontre certes des notations misogynes ponctuelles, mais qu'en conclure lorsque l'on constate qu'un autre poème de la même série présente le revers de la médaille - par exemple, si les organes génitaux féminins sont un « enfer » dans le sonnet 144, ils sont un « trésor » dans le sonnet 136 ?

Duncan-Jones n'est pas la seule critique dont le point de vue féministe l'amène à déformer le propos du sonnet 130. Felicia Jean Steele propose, pour sa part, une interprétation peu orthodoxe et peu satisfaisante du distique. À ses yeux, belied serait non pas un participe passé comme le croient les principaux commentateurs des Sonnets, mais un verbe au prétérit ; elle lit she comme un pronom renvoyant à la Dame et en conteste la substantivation (Booth glose she par "woman », p. 455). Elle reformule ainsi le dernier vers du poème : "As any [whom] she belied with false compare ». La Dame serait donc aussi remarquable que toutes celles dont elle-même a donné une fausse représentation, lecture qui fait d'elle un sujet plutôt qu'un simple objet de désir ( « [...] the dark lady may actually be a speaking subject rather than simply an object of visual desire [...]»). Cette proposition semble douteuse compte tenu de la teneur des douze premiers vers du sonnet, qui font clairement référence à une tradition poétique et non pas aux propos que la Dame aurait pu tenir sur d'éventuelles rivales. En outre, l'argumentation de cette critique manque de cohérence : d'une part, elle s'appuie sur le fait que Shakespeare ne substantive pas le pronom she dans les autres sonnets pour avancer que la construction du vers 14 est "strangely non-Shakespearian » (pourtant, ce pronom est substantivé dans Twelfth Night, I. v. 225) ; d'autre part, tout en reconnaissant que le relatif zéro était rare au XVI ${ }^{e}$ siècle (si on le trouve chez Sidney, comme elle le relève, n'est-il pas, lui aussi, bien peu shakespearien ?), elle n'hésite pas à postuler l'emploi d'un relatif zéro dans le dernier vers pour tenter d'étayer son interprétation du poème. Si sa démonstration peine à convaincre, c'est sans doute parce que sa conscience féministe l'amène à voir en la Dame une agente, une poétesse en puissance ( « an agent of misrepresentation and potentially a poet herself»), ce qu'elle n'est pas dans le sonnet 130. Voir Felicia Jean Steele, "Shakespeare's sonnet 130 », Explicator 62 (2003), p. 132-136. Pour rencontrer une femme poète capable de dissoudre la hiérarchie entre sujet masculin et objet féminin propre à la tradition pétrarquiste, il faudra attendre Lady Mary Wroth, nièce de Sir Philip Sidney et auteure d'Urania (1621), œuvre en prose incluant une série de sonnets intitulée Pamphilia to Amphilantus, que ses éditeurs Micah et Risa S. Bear commentent ainsi : " This project by itself stands on its head the Petrarchan tradition of courtly love poetry, for Amphilanthus [...] is not an object. He is instead enlisted in Pamphilia's quest for a mutually supported happiness founded upon the relinquishing of objectification, the mode by which oppressive power relations are constructed " (University of Oregon : Renascence Editions, 1996, n. p.).

82. Voici comment Paul Ramsey caractérise les sonnets relatifs à la Dame brune, The Fickle Glass. A Study of Shakespeare's Sonnets (New York: AMS Press, 1979), p. 8-9 : « They are, with two exceptions, poems about a black-haired woman and represent several attitudes toward her : (1) gay, gentle teasing $(128,130) ;(2)$ delicate tenderness $(143,145)$; (3) witty obscenity, with shades of self-contempt and 
tableau uniforme, on y découvre une mosaïque qui présente les états d'âme d'un sujet amoureux changeant, les intermittences du cœur et du corps, le dégoût que suscite l'asservissement par le désir, la tension entre raison et passion. Cette série de sonnets, comme le recueil dans son ensemble, est marquée par la discontinuité, l'ambivalence, la dissonance, aussi est-il vain d'y chercher une unité de ton ou de sentiment. Il n'est que de faire une lecture consécutive des sonnets 128,129 et 130 pour constater à quel point Shakespeare, comme Montaigne avant lui, peint le passage.

Déduire de la structure négative du sonnet 130 que la Dame n'est pas une beauté, c'est ne pas voir qu'à travers le portrait en creux que propose son auteur, c'est de l'art de représenter qu'il nous entretient : la métaphore poétique, aussi saisissante soit-elle, n'établit entre ses deux termes qu'un rapport arbitraire. D'autre part, les procédés s'usent - une figure trop employée perd sa fraîcheur et sa vigueur, aussi parle-t-on à bon droit de "métaphore morte " à propos de certaines expressions figées dans la langue. Cela dit, au lieu de se complaire dans une simple récusation des pratiques pétrarquistes, Shakespeare, plus subtilement, incorpore dans son poème les images mêmes dont il nie la pertinence, d'où la complexité de son « intextuation ${ }^{83}$ du corps féminin. Aussi faut-il parler à son sujet, non pas de sarcasme, mais d'hommage parodique. C'est l'ironie du retournement final qui en donne la plus éclatante illustration : alors que le poème tout entier dénonce la facticité des métaphores ou des comparaisons, il s'achève sur le mot compare après avoir recouru, précisément, à une comparaison - as rare as. L'ironie se trouve renforcée par l'emploi de l'adjectif rare, souvent utilisé par les sonnettistes élisabéthains pour qualifier leur Dame, comme c'est le cas d'Henry Constable dans le $4^{e}$ sonnet de la seconde décade de Diana ${ }^{84}$ (1592). En d'autres termes, la Dame brune shakespearienne

some hostility to her $(135,136,151)$; (4) anger, bitterness, hatred, thralldom $(131,133,134,137,138$, $139,140,141,142,144,147,148,149,150,152)$; (5) something between, or mixed of, teasing and the strong emotions $(127,132) »$.

83. L'expression est de Michel de Certeau, cité par Hunkeler : « Pars pro toto, totum in parte? Anatomie, amour et (dé-)composition à la Renaissance » in Variations 7 (2001), p. 71.

84. «Ladie in beautie and favour rare » dit son incipit. Voici celui du $25^{\mathrm{e}}$ sonnet de Delia de Daniel : " Raigne in my thoughts faire hand, sweet eye, rare voyce». Et celui du sonnet 18 de Chloris de William Smith : " My love, I cannot thy rare beauties place ». Dans le $38^{\text {e }}$ sonnet de Diella, Linche emploie rare dans une comparaison : "As a rare Jewell of most high esteeme " (v. 6). Le sonnet liminaire de Parthenophil and Parthenophe de Barnes présente la Dame comme " Of all women rarest !" (v. 2). 
a autant de charme que toutes celles qui sont glorifiées dans les recueils de sonnets contemporains, mais sa glorification passe par le mépris que le Poète feint d'éprouver à l'égard du répertoire d'images rebattues où puisent les pétrarquisants. En faisant mine de congédier le false compare, le sonnet opère un déplacement des références : la soumission à une norme descriptive cède la place à une subjectivité assumée ${ }^{85}$ - le «I think » du vers 13 vient couronner une succession de verbes à la première personne - , subjectivité dont on peut rappeler qu'elle est l'un des traits du blason tel que Marot le concevait.

\section{๙}

Le sonnet 130 n'est donc pas un contreblason stricto sensu, un contreblason marotique : comme l'indique clairement son distique, l'image de la Dame n'y est nullement ternie - ce sont les codes qui régissent la représentation pétrarquiste de la Dame qui font l'objet d'un questionnement amusé.

En somme, imaginer que le poète tourne sa maîtresse en dérision en soulignant ses innombrables défauts, c'est se tromper de paradigme : il brosse moins le portrait d'une femme que d'un genre poétique ; ce n'est pas la Dame qu'il met à nu, c'est le blason. Contreblasonner, en l'occurrence, ce n'est pas blasonner à rebours ${ }^{86}$, c'est mettre en lumière les dessous du blason, c'est construire un subtil métablason.

Par conséquent, le tour de force que réussit Shakespeare dans le sonnet 130 consiste à proposer une parodie du blason du corps féminin tout en maintenant l'éloge de la Dame propre au blason et, plus généralement, à la tradition pétrarquiste dont il se démarque non sans l'avoir parfaitement intégrée. C’est ce

\footnotetext{
Watson introduit le poème 34 de son Hekatompathia de cette façon : "The Author in this Sonnet very highly commendeth the most rare excellencies of his mistress, avouching her to have no equal "; à l'instar de Constable, il associe rare et beauty dans le poème 48 du même recueil. Ces quelques exemples suffisent à infirmer l'argument de Pointner selon lequel, si Shakespeare n'a pas préféré l'adjectif fair à rare, c'est pour indiquer que sa Dame est tout sauf belle (Pointer, p. 85 : "She is anything but beautiful - Shakespeare could have substituted "rare" of line thirteen by "fair" without even distorting the rhyme $[\ldots] »)$.

85. Ce thème est développé dans le sonnet 131, où le Poète, contre ceux qui ne verraient pas la Dame d'un oil aussi favorable que lui, affirme : « Thy black is fairest in my judgment's place ».

86. Marot emploie l'expression « Ains que chascun le Rebours chanter vueille » dans l'épître "A ceulx, qui apres l'Epigramme du beau tetin en feirent d'aultres », Euvres poétiques I, p. 338.
} 
double mouvement d'appropriation et de distance mutine qui fait la singularité de sa voix poétique. ${ }^{87}$

87. Une étude comparative de 22 traductions françaises de ce sonnet fait l'objet d'un autre article, à paraître dans « Cahiers Shakespeare en devenir ». 